\title{
A Study of Exercise Intensity Based on Individual's BMI and Heart Rate
}

\author{
Xiaoao Zou1, Junhui Gao ${ }^{2 *}$ \\ ${ }^{1}$ The Village School, Houston, TX, USA \\ ${ }^{2}$ American and European International Study Center, Wuxi, China \\ Email:*jhgao68@163.com
}

How to cite this paper: Zou, X.A. and Gao, J.H. (2018) A Study of Exercise Intensity Based on Individual's BMI and Heart Rate. Health, 10, 902-906.

https://doi.org/10.4236/health.2018.107066

Received: June 9, 2018

Accepted: July 1, 2018

Published: July 4, 2018

Copyright (c) 2018 by authors and Scientific Research Publishing Inc. This work is licensed under the Creative Commons Attribution International License (CC BY 4.0).

http://creativecommons.org/licenses/by/4.0/

\begin{abstract}
The purpose of this research is to explore if individuals of different BMI can achieve a given intensity using the same exercise load. For the first part of our research, a survey was sent out to gather responses from people of various age groups. We asked their weight, height, and resting heart rate. We found out there was no correlation between BMI and heart rate. We also designed an experiment to study the relationship between BMI and exercise intensity. The result shows people with higher BMI need more exercise load in order to match the exercise intensity of people with lower BMI.
\end{abstract}

\section{Keywords}

BMI, Heart Rate, Exercise

\section{Introduction}

In recent years, more and more people get motivated to go to the gym and start working out. A lot of people are willing to invest time and money to improve their general health and fitness. Newly-built gymnasiums, weight rooms, and swimming pools also facilitate people's activities. It's not surprising to see people in many developing countries to pay more attention to their body. However, every formation a habit like exercising needs a learning process. New vocabularies thus come into our sight.

Some of the words that will be discussed in the research are BMI (Body Mass Index) and heart rate. The measurement of BMI is relatively simple. You will need to know a person's body weight in kilograms and then divide it by the square of his or her height in meters. It's a convenient way to categorize a person into underweight, normal weight, or obesity [1]. BMI is commonly used in sta- 
tistical studies. The second word, heart rate, means the speed of heart contractions (usually measured by how many beats in a minute). For a healthy adult, the normal range of resting heart rate is from 60 to 100 beats per minute. People who have been exercising regularly tend to have a lower number because their heart muscle is trained to pump more oxygen-rich blood into the body. Heart rate is widely used to calculate exercise intensity, which is personalized depending on the goal of an individual. Some people want to increase strength. Some want to lower their body fat percentage. For example, setting up a targeted heart rate is important in aerobic exercises. Maintaining the targeted heart rate for a period of time (20 minutes minimum) will efficiently enhance a person's cardiovascular health [2].

In this research, we will try to implement BMI into consideration when we study the relationship between heart rate and intensity.

\section{Results}

Our first conclusion is: a person's BMI has no correlation between his or her resting heart rate.

Our second conclusion is: a person with a higher BMI need more exercise load in order to achieve the same workout intensity.

\section{Materials and Methods}

Our research is composed of two parts. The first part is a survey, which is used to determine the correlation between BMI and heart rate. The second part is a study on BMI and exercise intensity. The study is based on a theory that states exercise intensity can be represented by the change in heart rate.

\subsection{Relationship between BMI and Heart Rate}

We designed an online survey, asking respondents about their height, weight, resting heart rate, age, and sex. The results of the survey from 343 respondents are collected by WenJuan Net [3]. We calculated their Body Mass Index and investigated the correlation between BMI and heart rate. We represented the data (BMI and heart rate) using linear regression. The coefficient $r$-squared is 0.0095 , showing virtually no correlation. Figure 1 shows most BMI measurements are located around $20-21$.

\subsection{Relationship between BMI and Change in Heart Rate}

Previous experiments showed that BMI was not significantly related to heart rate. To this end, we have designed an experiment to explore whether different BMI people need the same intensity of exercise. We believe that the intensity of exercise can be characterized by the change of heart rate before and after exercise. We found 10 people, divided into 2 groups, 5 in each group, and the average BMI of the two groups was significantly different. Let 10 people run 200 meters at the fastest speed to calculate the change of heart rate before and after 


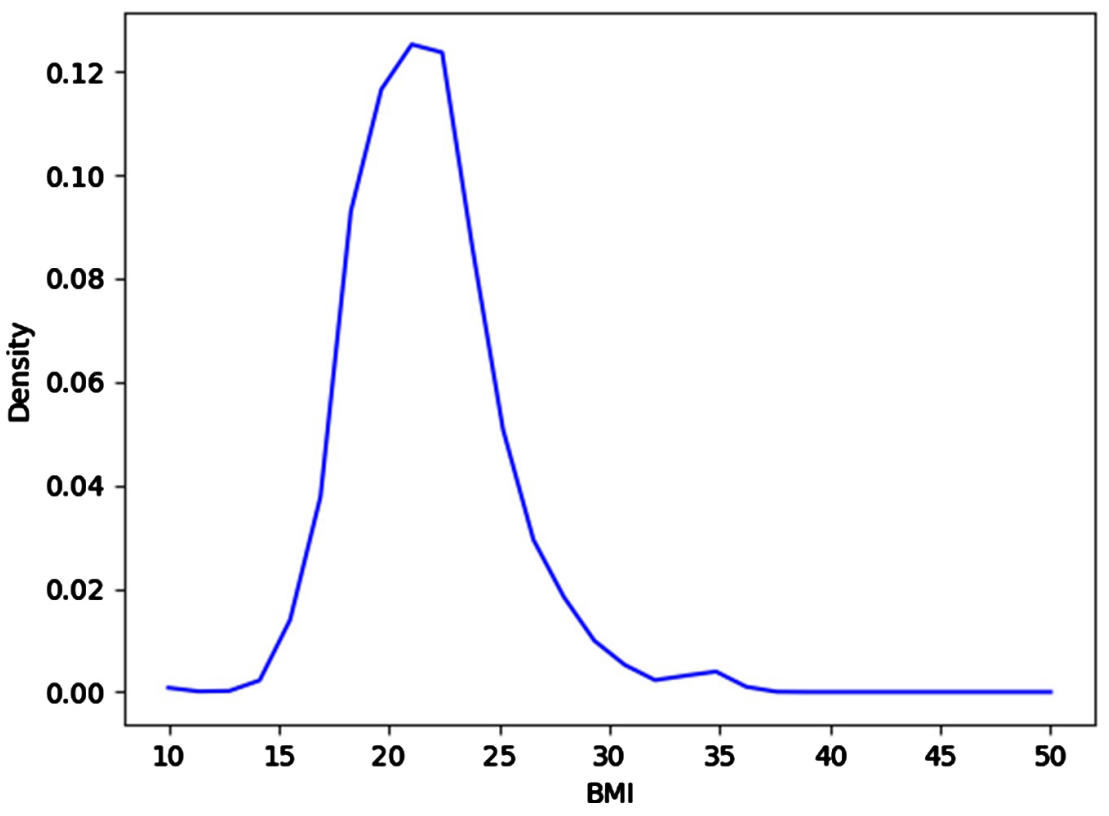

Figure 1. The distribution of BMI.

Table 1. Heart rate changes before and after exercise in two groups.

\begin{tabular}{cccccccc}
\hline & Height & weight & HR1 & HR2 & Age & BMI & Change in HR \\
\hline A1 & 1.72 & 65 & 66 & 156 & 18 & 21.97 & 90 \\
A2 & 1.71 & 63 & 66 & 210 & 18 & 21.55 & 144 \\
A3 & 1.75 & 61 & 78 & 168 & 17 & 19.92 & 90 \\
A4 & 1.75 & 62 & 60 & 204 & 17 & 20.24 & 144 \\
A5 & 1.71 & 62 & 54 & 150 & 17 & 21.20 & 96 \\
Avg. & & & 64.8 & & & 20.98 & 112.8 \\
B1 & 1.75 & 103 & 84 & 171 & 18 & 33.6 & 87 \\
B2 & 1.94 & 107 & 78 & 168 & 18 & 28.4 & 90 \\
B3 & 1.88 & 100 & 86 & 168 & 17 & 28.3 & 82 \\
B4 & 1.75 & 105 & 78 & 172 & 16 & 34.3 & 94 \\
B5 & 1.85 & 115 & 84 & 180 & 16 & 33.6 & 96 \\
Avg. & & & $\mathbf{8 2}$ & & & 31.64 & 89.8 \\
\hline
\end{tabular}

running. The results are shown in Table 1.

The average BMI of group A was 20.98, and group B was 31.64. Obviously, there was a significant difference between the two groups of BMI. Before and after exercise, the average heart rate in group A was 112.8, while that in group B was 89.8 . This shows that in order to achieve the same exercise intensity (heart rate change), people with high BMI need more exercise.

\section{Applications}

Understanding how to use BMI and heart rate to design workout intensity can help people exercise scientifically and avoid mistakes. 
First of all, Chinese students nowadays don't get an adequate amount of daily exercise. It's partially due to the lack of science in their Physical Education class. For instance, students in most middle schools are required to run 1000 meters at the beginning of every P.E. class. It's a simple task for students who have a lower BMI and exercise regularly. Since they pass the running test easily, P.E. teachers will give them permission to other activities of their choice. In other words, teachers don't expect more from them. In my opinion, the absence of progressive overload decreases the exercise intensity in those students. They don't have to work hard to make change to their body since they are already accustomed to this intensity. Therefore their potentials are limited. If P.E. teachers could design a progressive intensity for those individuals, We believe they would witness significant improvements.

Secondly, commercial gyms and personal trainers can use this knowledge to better help their clients. The goals of gym goers can be categorized to fat-loss, gaining muscle, or gaining strength. When trainers are making workout plans, they can refer to the trainees' BMI, resting heart rate, and age. For people who want to lose weight, the fat burning zone is at $60 \%-70 \%$ of their max heart rate [4]. After achieving the targeted heart rate, trainers can adjust the time depending on trainees' BMI.

\section{Discussion}

Before we started our research, an online journal called Exercise and associated dietary extremes impact on gut microbial diversity [5] caught our eyes. This journal discusses how the gut microbiota of elite athletes differ from those of normal participants. The researchers of this journal have proved that exercising can enhance the biodiversity of human microbiota, by analyzing data collected from athletes and their control groups. Nevertheless, we believe that the experiment designing still requires a bit more adjustment to be considerate. The researchers kept the exercise intensity of two control groups (Group 1. BMI $\leq 25$, Group 2. BMI $>28$ ) nearly equal. The exercise intensity is represented by creatine kinase levels. However, they did not realize BMI could influence one's exercise load, even when the intensity is same. As a result, our research could provide more information in this topic.

In general, the higher the heart rate, the more intense the movement. When it comes to measuring exercise intensity, Max Heart Rate is an indicator that cannot be ignored. Using the formula "maximum heart rate = 220-age", the maximum heart rate can be roughly calculated [6]. For example, Kai is an 18-year-old high school student and his maximum heart rate is about 202. If Kai wants to perform aerobic exercise with an intensity of $80 \%$, his target heart rate can be set at $202{ }^{\star} 80 \% \approx 162$ beats/minute (maximum heart rate ${ }^{\star}$ exercise intensity) according to the Maximal heart rate method. Another method of determining the target heart rate introduces and uses heart rate reserve, which is the value of the highest heart rate minus the resting heart rate. This method was proposed by the 
Finnish doctor Martti Karvonen in 1957: target heart rate $=$ training intensity ${ }^{*}$ heart rate reserve + resting heart rate. Kai first measured his resting heart rate of 76 beats/minute. According to the formula, his target heart rate $=80 \% \times(202-$ $76)+76$. The result is about 177 beats/minute [7]. However, we find that this method is not perfect. For example, height and weight of the subject are neglected.

\section{Conclusion}

In our research, we first verified through a survey that there is no direct relationship between heart rate and BMI. With the clear link between heart rate and exercise intensity, we conducted experiments to further explore the relationship between BMI and exercise intensity. We summoned 10 students of the same age, collected their weight, height, and heart rate at rest, and divided them into two groups with 5 individuals each by their BMI. We recorded the heart rate of these two groups of students after intense exercise. After analyzing the data, we concluded that people with higher BMI need more exercise to achieve the same level of intensity. We hope that our research results can bring practical applications to people who care about their health.

\section{Acknowledgements}

We are grateful to Ms. Zhang and Mr. Yang of Jiangsu Tianyi High School for helping with collecting data. We also want to thank the 10 students who partook my experiment. Thank you all for your time.

We hope to see our readers gain some insight from this research. We would achieve our goal if you readers could learn something new.

\section{References}

[1] Nuttall, F.Q. (2015) Body Mass Index Obesity, BMI, and Health: A Critical Review. Advances in Pediatrics, US National Library of Medicine. www.ncbi.nlm.nih.gov/pmc/articles/PMC4890841/

[2] Know Your Target Heart Rates for Exercise, Losing Weight and Health. American Heart Association.

https://healthyforgood.heart.org/move-more/articles/target-heart-rates

[3] https://www.wenjuan.com/

[4] Achten, J., Gleeson M. and Jeukendrup, A.E. (2002) Determination of the Exercise Intensity That Elicits Maximal Fat Oxidation. Medicine and Science in Sports Exercise, 34, 92-97. https://doi.org/10.1097/00005768-200201000-00015

[5] Clarke, S.F., et al. (2014) Exercise and Associated Dietary Extremes Impact on Gut Microbial Diversity. Gut, 63, 1913-1920. https://doi.org/10.1136/gutjnl-2013-306541

[6] Target Heart Rate and Estimated Maximum Heart Rate. Centers for Disease Control and Prevention, Centers for Disease Control and Prevention, 2015. https://www.cdc.gov/physicalactivity/basics/measuring/heartrate.htm

[7] Kelly, M. Advances in Aerobic Training: How to Apply the New Heart Rate Formulas. ACE.

https://www.acefitness.org/education-and-resources/professional/expert-articles/35 $\underline{02 / \text { advances-in-aerobic-training-how-to-apply-the-new-heart-rate-formulas }}$ 